\title{
Editorial
}

\section{Milestones in OR}

Journal of the Operational Research Society (2009) 60, S1-S4. doi:10.1057/jors.2009.15

A very popular (and cheap) formula for a Saturday night TV show is 'Your top $100 \ldots$..'-movies, albums, comedy shows, sporting moments, and so on. Inevitably, the viewer is left wondering who on earth chooses these lists, and on what basis do they decide on the ranking order? People can argue about this sort of thing endlessly (and pointlessly) - usually, over a pint or two in the pub. As editors of this Special Supplement, we have some sympathy with the makers of such programmes. There was no obvious rationale to our choice of papers. We did not systematically decide on a set of key topics in operational research (OR) and then commission papers on these subjects, as we would have done had we been editing a textbook. Instead, we simply invited many well-known authors-mainly, but not exclusively, British, and both academics and practitioners-to present keynote talks based loosely on the theme of 'Fifty years of...' at the OR Society's Golden Anniversary conference, held at the University of York in September 2008. We then encouraged them to write up their talk as a paper for the Conference Keynote Papers and/or this JORS Anniversary Supplement. From the (record) set of 29 Keynote talks at the conference, 15 papers have made their way through the standard JORS refereeing process into this collection. The result is a somewhat arbitrary and indeed idiosyncratic set of papers. We are aware that there are many obvious gaps_-not the least glaring of which is mathematical programming!-which a truly representative survey of ' 50 years of OR' would clearly have had to contain. Equally, there are papers on subjects which some people might not see as mainstream OR at all. We make no apology for this, but simply hope it might stimulate a few pub conversations and arguments! We hope you enjoy reading the papers as much as we have enjoyed putting this collection together.

People have tackled our 'Fifty years of ...' theme in different ways. Some have taken a fairly literal approach and have identified key papers decade by decade. Others have written tutorials which present a very useful introduction to their subject. Others have adopted a more discursive approach, taking the opportunity to reflect more generally on developments, or even missed opportunities, in a particular technique or application area. We start with Rosenhead's overview and end with Royston's look towards the future, but otherwise have organized them with all the technique-based papers first and the application-based papers second, and within these categories, in alphabetical order by name of first author-and occasionally, we discuss whether the topic would have appeared in an OR textbook in 1958. We are sure that all these papers will be widely read (and referenced!) in future. Overall, this collection of papers provides a fascinating insight into a range of topics, and will serve as a worthy legacy from the OR50 conference.

In our first paper, Rosenhead tackles one of the most difficult tasks of any author-to write a single journal article to reflect on 50 years of an entire field. He approaches his 'Reflections on Fifty Years of Operational Research' with the pragmatic view that such an article will inevitably be selective and so he focuses on the milestones that have punctuated the last 50 years of OR. He takes the reader on the journey through the origins of OR in the Second World War, its place in the post-war reconstruction and contribution to the economic boom, the shifting philosophy of OR between problem-focused and technique-focused, OR's movement in and out of favour with industry and funders, 'The Ackoff Papers' and their ripples/waves across the UK, and the Thatcher and Reagan years. While taking a UK perspective, Rosenhead lightly explores the different experiences of OR in other countries, most notably USA, suggesting that UK tradition of OR is distinctive with strengths (eg the link between theory and practice) but also weaknesses (eg lesser focus on technical creativity). This is an ambitious topic for any author but Rosenhead, as part of the history of the last 50 years, provides a reflective account which does the field immeasurable justice.

Data mining is an example of a topic which would not have been included in an OR textbook 30 years ago, but is now widely used in many application areas, including credit risk, marketing, fraud detection and counter-terrorism. Baesens et al provide a very clear introduction to the topic. Simply stated, the aim of data mining is to extract interesting patterns from data. Automated data collection approaches such as barcoding and RFID enable vast data sets to be available for analysis, as anyone who uses a loyalty card in a supermarket knows. Modern data mining techniques are based on a wide variety of OR approaches. Many challenges still need to be tackled, ranging from data quality issues to the problem of how to include domain experts' knowledge, and Baesens et al's paper concludes with a series of upcoming trends and challenges for data mining and its role within OR.

Next, Jackson dissects 'Fifty years of systems thinking for management' using Boulding's hierarchy of complexity to explore the emergence and development of applied 
systems thinking. By showing the closeness of OR and systems thinking the paper attempts to raise awareness of the relevance of systems thinking for OR theory and practice, with the aim of bridging a gap of the camps knowing 'surprisingly little about the other'. Jackson challenges the OR reader to reconsider the view of systems thinking as being 'unscientific, or impractical and too much in love with philosophizing' by offering an argued analysis of the relationship between the camps that aims for OR readers to reconsider their image of systems thinking and its relationship with OR.

Morton and Phillips represent a British viewpoint of reasoning about uncertainty in their paper titled 'Fifty Years of Probabilistic Decision Analysis: A view from the UK'. They show that a range of techniques were developed in Britain for thinking quantitatively about uncertainty and they show how these techniques enabled the field to move from theoretical appreciation to a practical solution. However, they believe that recent US-focussed debates on the topic have not reflected the rightful prominence of British researchers and their contribution to advancing the field. Consequently, their paper aims to rebalance the debate by positioning this body of British research in a wider context.

Scheduling is one of the most widely researched areas within OR and although arguably this field of research can be traced back to the early 20 century, Potts and Strusevich in their paper survey some of the landmark papers in deterministic scheduling from the mid-1950s. The authors highlight the key contributions that have shaped the field with particular focus on scheduling problems that arise in production industries. Potts and Strusevich observe that considerable progress has been made with contributions by researchers from varied disciplines (eg, mathematics, OR/MS computer science, engineering and economics) and that although the area is mature with many theories and techniques that can be used to tackle complex problems, many challenges remain for new and established researchers.

A somewhat critical, even slightly negative view of the success of an OR approach is adopted by Taylor $e t a l$ in their paper on simulation. No textbook on OR would be complete without a chapter on simulation, although traditionally this would be at the very end of the book and might have the (implicit or explicit) subtitle 'when all else fails...'. Nevertheless, the fact remains that simulation is probably the most widely used OR approach in practice. Taylor et al present a review of the simulation modelling literature from 2000 to 2005. They found a relative lack of 'real-world' involvement in simulation modelling research and an even greater lack of evidence of 'real-world' benefit, arguably very alarming outcomes for an applied field. Taylor et al ask if this is the natural evolution of a field that has existed for half a century, or an indication of a worrying problem, and discuss whether or not simulation modelling research urgently needs to face a 'reality check'.
Queueing theory is one of the longest established modelling approaches available to operational researchers, with its origins in the telecommunications sector, and would have certainly featured in a survey of OR techniques 50 years ago. In his paper, Worthington purposefully uses the term 'queueing models' to both emphasize the application rather than just the theory, and to indicate modelling approaches in general which are appropriate for tackling queueing problems, and not exclusively just those based on queueing theory. The resulting paper is a comprehensive review of modelling approaches and applications based around what Worthington proposes as five dimensions: single server versus multi-server versus infinite server; exponential versus non-exponential; steady state versus time-dependent; single node versus tandem queues versus networks of queues; and extra features such as queueing system rules and behavioural factors. The paper finishes by identifying some possible future research and development themes. This paper will be of great interest to both experienced researchers in this field as well as those wishing to gain an appreciation of modelling approaches for tackling queueing problems and their applications.

Cutting and packing problems have been a core area of OR for many decades. Bennell and Oliveira are two of the bestknown researchers in a particular (and more recent) variant of these problems, irregular shape packing. An everyday example of this problem arises in the garment manufacturing industry, where irregular-shaped pattern pieces have to be cut from a length of cloth, with the aim of minimizing waste. As with all combinatorial problems, the past five decades have seen enormous advances in the size and complexity of problems which can be solved, due to both computer power and algorithmic sophistication. This paper focuses on algorithmic developments in irregular shape packing, but is not a chronological account or an exhaustive review. The authors present a very readable introduction to the topic, drawing on the literature to describe and evaluate the core approaches, which are mainly heuristic, with a few notable exceptions. Bennell and Oliveira explore different ways of representing the problem and mechanisms for moving between solutions, and discuss the future challenges for researchers in this area.

The term 'Asset Management' first arose in the finance sector with its core component being the trade off between risk and return. Since then, the approaches, techniques and tools developed have been adapted to suit Asset Management practices in other industries, such as electricity, gas and water, and it is these later industries that provide the focus of the review paper by Brint $e t$ al. The paper first examines what is meant by Asset Management and concludes that it is the integration of among other things, maintenance and replacement analyses, finance, economics and systems engineering. The paper then describes three factors that have driven recent developments: the ageing of infrastructure, the advent of new technology systems and changes in the structure of the industries. The authors highlight the current provision of the field and the modelling techniques adopted, before 
considering possible futures and where OR can play a significant role.

One of the key features of British OR (as identified by the EPSRC International Review of OR Research, 2004) is the strength of 'soft' OR. This is an aspect of OR which is still regrettably ignored by many standard textbooks, although its origins are well over 50 years old. Edwards et al present a paper which reflects on the field of Knowledge Management $(\mathrm{KM})$, a topic which was unheard of 30 years ago. They argue that although both the UK and US OR Societies publish KM journals, in reality OR has not played a major role in the development of KM and has not (as yet) achieved its potential. Despite this disappointing performance to date, they argue that OR is uniquely placed to lead future KM developments, and suggest that a limiting aspect of whether OR will be seen to have a significant profile will be the extent to which developments are recognized as being informed by OR.

Another of the topics which would definitely have been included in a 1958 textbook is locational analysis, an interdisciplinary field rooted in mathematics, computer science, OR, economics, geography and other areas. Location theory concerns the placing of 'facilities' in a spatial domain, and is a widely researched field. Smith et al do not present a complete survey of the topic, but aim to give the reader a concise but representative selection of key results. They argue that locational analysis has grown to maturity over the last decades, from its earliest roots, to fruitfulness in a wideranging number of strands that join with other disciplines and applications areas. The paper charts the progress of location theory in three stages: a period of early contributions, when a number of geometrical and geographical problems were studied; a 'coming of age' with the development of fundamental, classical problems; and a third period of new models and new applications.

The 50 years theme continues in Simpson and Hancock's review of 'Fifty years of operational research and emergency response'. The authors address an important topic and show the strong impact which OR has made to the field of emergency preparedness and response. However, they find that the problems that have been addressed by OR are those that have more structure, concluding that resilience is less structured than these examples and so OR will need to rise to the challenge of dealing with much more unstructured problems if it is to continue to have impact on practice in this field. Interestingly, they use a novel approach to analysing the literature and presenting their findings. As this approach is based on OR, and could be easily transferable across OR and beyond, the paper will also attract readers who seek interesting ways to work with literature.

The topic of forecasting and planning for inventory management is reviewed by Syntetos et al who take their perspective from divergent strands of the literature, namely: system dynamics, control theory and forecasting theory. The resulting paper provides a comprehensive overview of the topic and the variety of methodologies adopted. The authors observe that this topic has received considerable attention from the OR community over the last 50 years because of its implications for decision making, both at the strategic level of an organization and at the operational level. However, they comment that although the plethora of different perspectives to the same problem may be perceived as a healthy development in terms of knowledge advancement, it also signifies the fragmentation of OR into sub-disciplines that are not adequately cross-informing theory and practice, and that inter-disciplinary opportunities for inventory forecasting and planning have not been adequately addressed. The authors express hope for the next 50 years given the many opportunities for further research and for a healthy cross-utilization of ideas.

One of the many interesting questions Wright discusses in his highly entertaining yet erudite review of OR in Sport, is which OR academics' names are best known to the general public. The answer is almost certainly Frank Duckworth and Tony Lewis, whose method for settling the outcome of rainaffected cricket matches has been universally adopted by all cricketing nations and whose initials now appear on cricket scoreboards all over the world. Wright argues that 'the phrase 'duckworth/lewis' will soon become lower-case like sellotape, biro or hoover-proper names that are now regarded by many as a part of the English language'.

For our final paper of this Special Supplement, we have chosen Royston's paper on OR in health, since much of what Royston says about the future of OR in this field is true for OR in general. The paper draws on the author's 30 years of experience working in this area, having just retired as Head of Strategic Analysis and Operational Research within the Department of Health. The paper charts the contribution of OR at local and national levels since the creation of the NHS, which itself celebrated its Sixtieth Birthday in 2008. Despite the undoubted contribution of OR in this area, which was identified along with 'soft' OR as a key strength of British OR in the 2004 EPSRC International Review, Royston raises some concerns, such as the lack of visibility and a poor rate of implementation, that must be addressed. The paper then looks to the future and highlights some of the challenges that healthcare faces and the corresponding opportunities for OR. Royston ponders how OR can meet these challenges. He suggests we should remember some wise words from Blackett who published wartime principles for effective OR (Blackett, 1948) that included: 'Collaborative', 'Grounded' and 'Pathfinding'. Royston concludes by suggesting five key areas which are likely to determine effectiveness. These are certainly not restricted to health OR but surely are guiding principles for OR in general, in order that our discipline may flourish over the next 50 years.

In conclusion, we would like to thank all the contributors to this issue, but we would also like in particular to thank the reviewers, who did a top-quality job in remarkably little time. We are tempted to paraphrase Churchill and say 'Never in the history of OR has so much refereeing been done so quickly 
with such expertise'. The speed and quality of the reviews was truly impressive, as was the collegial spirit with which people agreed to do them, and suggests that the next 50 years of OR (and the next 60 years of JORS) will be in safe hands.

\section{References}

Blackett PMS (1948). Scientists at the operational level. Advancement of Science 5: 30-38.
Review of the Research Status of Operational Research in the UK (2004). EPSRC/ESRC, Swindon, UK. Downloadable from www. epsrc.ac.uk/AboutEPSRC/IntRevs/2004ORIR/review.htm, accessed 4 February 2009.

University of Southampton, UK

Cardiff University, UK

Sally Brailsford

Paul Harper

Aston University, Birmingham, UK 